\title{
Bit Error Rate Evaluation of IEEE 802.16 (WiMAX) in OFDM System
}

\author{
Hardeep Kaur \\ Department of Electronics Technology \\ Guru Nanak Dev University
}

Amritsar

\author{
M.L Singh \\ Department of Electronics Technology \\ Guru Nanak Dev University \\ Amritsar
}

\begin{abstract}
This paper investigates several modulation techniques for IEEE 802.16 (WiMAX) based OFDM system including BPSK, QPSK, 8PSK and various levels of QAMs. The system performance is gained by dynamically choosing the modulation technique based on the channel conditions.
\end{abstract}

The performance of OFDM system is accessed by using computer simulations performed using MATLAB 7.3. In this paper, transmitter and receiver models are simulated according to the parameters established by the standard, to evaluate the performance. Also, convolution coding is used to improve the system performance.

Use of adaptive modulation can effectively control the BER of the transmission, as sub carriers that have a poor $\mathrm{E}_{\mathrm{b}} / \mathrm{N}_{\mathrm{o}}(\mathrm{db})$ can be allocated a low modulation scheme such as BPSK. For good $\mathrm{E}_{\mathrm{b}} / \mathrm{N}_{\mathrm{o}}(\mathrm{db})$, the high modulation scheme giving priority to spectral efficiency can be considered. $\mathrm{E}_{\mathrm{b}} / \mathrm{N}_{\mathrm{o}}$ of the channel is estimated before the transmission. The modulation scheme is set based on the SNR of the channel.

\section{General Terms}

Coding data rate, Interleaving, additive white Gaussian Noise, Bit Error Rate, Adaptive Modulation, channel conditions etc.

\section{Keywords}

OFDM (Orthogonal Frequency Division Multiplexing),BPSK (Binary Phase Shift Frequency),QAM (Quadrature Amplitude Nodulation),BER(Bit Error Rate),SNR(Signal to Noise Ratio).

\section{INTRODUCTION}

In recent years, orthogonal frequency-division multiplexing (OFDM) has emerged as the standard of choice in a number of important high-data-rate applications. In OFDM, instead of using a single wide-band carrier to transmit information, a large number of parallel narrow-band sub-carriers are used. OFDM is very effective in dealing with multipath fading. But it is very sensitive to frequency offset and phase noise. It is adopted by various standards like DSL, 802.11, DAB, DVB etc. In OFDM serial-to-parallel transmitter converts the incoming high-rate data stream into low-rate streams, then transmits each low-rate data stream over a unique orthogonal carrier. The data rate of each transmitted stream is effectively reduced by a factor of from the original data rate. Utilizing this strategy, OFDM drastically reduces intersymbol interference (ISI) by avoiding multipath in frequencyselective channels. [1]

In coded OFDM (COFDM), a rate $(\mathrm{k} / \mathrm{n})$ coder followed by an interleaver is added prior to serial-to-parallel conversion. This effectively allows each symbols $\mathrm{k}$ to be sent over distinct frequencies. This introduction of redundancy and frequency diversity overcomes much of the signal degradation due to fading. The downside of such a coding is reduced throughput [by a factor of $n / k$ ].[2]. WIMAX is short form of e Worldwide Interoperability for Microwave Access. It is a $4 \mathrm{G}$ technology defined in IEEE 802.16 standards.[4,6]WiMAX is expected to provide fixed, nomadic, portable and mobile wireless broadband connectivity without the need for direct line-ofsight (LOS) with a base station. [5] It has many types of highbandwidth applications.In this paper, a model of an OFDM system with IEEE 802.16 implementation is developed using simulation in Matrix laboratory language (MATLAB) on which BER calculations for various digital modulation schemes like BPSK, QPSK, 8PSK,various levels of QAMs are carried out. The convolution coding and interleaving is applied to improve BER performance of OFDM system. The OFDM signal is transmitted over the AWGN channel for various signal-to-noise ratio (SNR) values. To evaluate the performance, for each SNR level, the received signal was demodulated and the received data was compared to the original information. The result of the simulation is shown in the plot of the bit error rate versus $\mathrm{E}_{\mathrm{b}} / \mathrm{N}_{\mathrm{o}}$, which provides information about the system's performance.

\section{Block Diagram of OFDM}

To implement the OFDM transmission scheme, the whole design is divided into three sections -Transmitter, Channel and Receiver.

In the transmitter, binary input data sequence is taken. Forward Error-Correction Coding (FEC) and interleaving is done to provide frequency diversity. The sequence is encoded by a convolutional encoder. Then Interleaving is applied to randomize the occurrence of bit errors prior to increase performance. After interleaving, the binary values are converted to symbol values, on which digital modulation scheme is applied. Previously, multi-carrier systems were implemented through the use of separate local oscillator. This was both inefficient and costly. With the advent of cheap 
powerful processors, the sub-carriers can now be implemented by the FFT which keep tones to orthogonal with each other.[2]

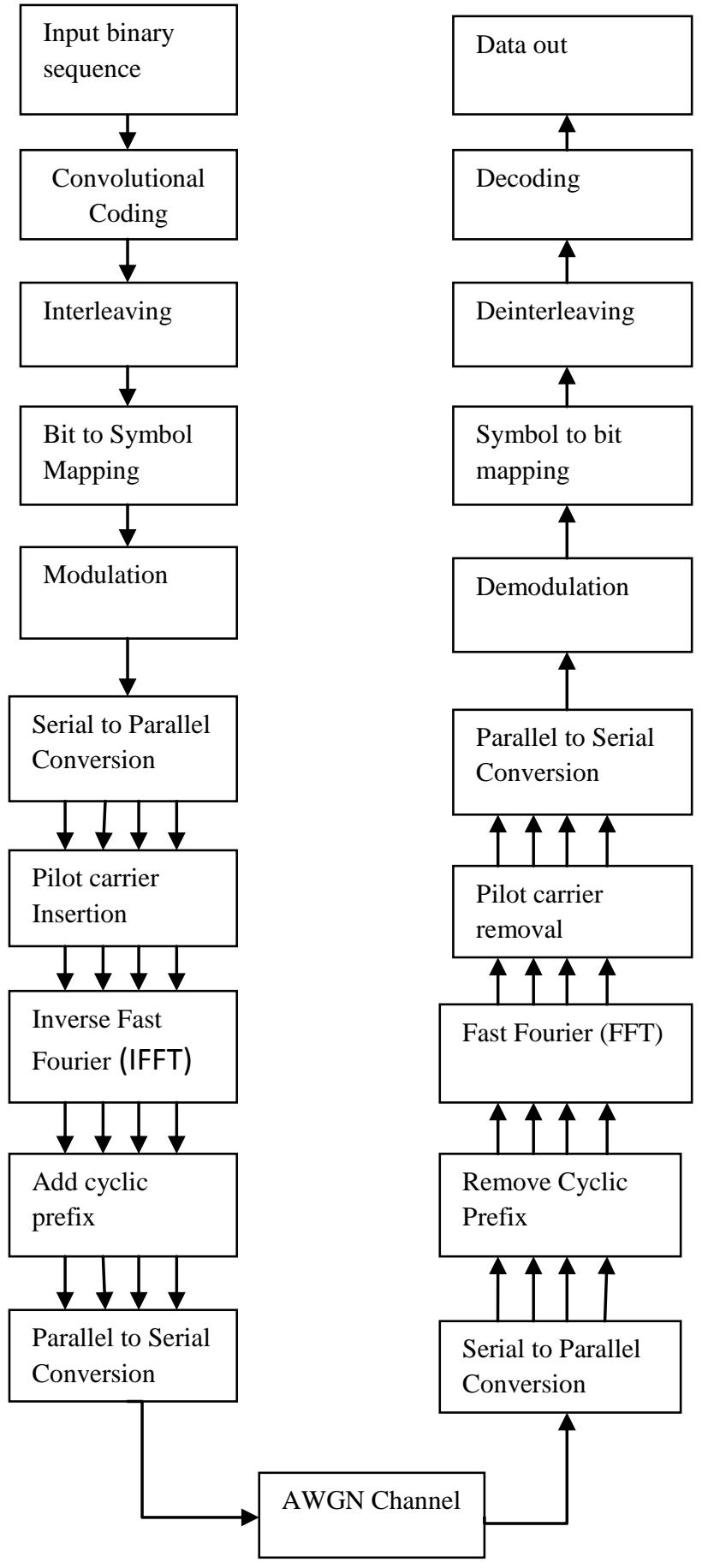

Fig. 1: Block diagram of OFDM system

To implement the OFDM transmission scheme, the whole design is divided into three sections-Transmitter, Channel and Receiver.

In the transmitter, binary input data sequence is taken. Forward Error-Correction Coding (FEC) and interleaving is done to provide frequency diversity. The sequence is encoded by a convolutional encoder. Then Interleaving is applied to randomize the occurrence of bit errors prior to increase performance. After interleaving, the binary values are converted to symbol values, on which digital modulation scheme is applied. Previously, multi-carrier systems were implemented through the use of separate local oscillator. This was both inefficient and costly. With the advent of cheap powerful processors, the sub-carriers can now be implemented by the FFT which keep tones to orthogonal with each other.[2]The symbol is modulated onto sub carriers by applying the Inverse Fast Fourier Transform (IFFT). The output is converted to serial and a cyclic extension is added to make the system robust to multipath propagation. In channel, additive white Gaussian noise characteristics are taken. The receiver performs the reverse operations of the transmitter. After removing the cyclic extension, the signal can be applied to a Fast Fourier transform to recover the modulated values of all subcarriers. The modulated values are then demapped into binary values, and finally deinterleaving and Viterbi decoder decodes the information bits.

\section{Simulation Results}

The simulations are performed on following standard parameters:

Table 1: IEEE 802.16 Parameters consider in simulation

\begin{tabular}{|l|c|}
\hline \multicolumn{1}{|c|}{ Parameters } & Values \\
\hline Number of OFDM symbols & 10000 \\
\hline Total data & 960000 \\
\hline Number of bits per OFDM symbol & 96 \\
\hline Number of data sub-carriers & 96 \\
\hline $\begin{array}{l}\text { Number of data sub-carriers after } \\
\text { coding }\end{array}$ & 192 \\
\hline Number of FFT points & $\begin{array}{c}\text { 256 } \\
\text { Cyclic prefix }\end{array}$ \\
\hline OFDM symbol & $\begin{array}{l}\text { BPSK, QPSK, } 8 \\
\text { PSK,QAMs }\end{array}$ \\
\hline Modulation scheme & $\begin{array}{l}\text { Convolutional, } \\
\text { code } \\
\text { rate } 1 / 2, \\
\text { ponstraint length } \\
\text { generator }\end{array}$ \\
\hline Coding & $171,133]$ \\
\hline
\end{tabular}

Case1: Considering BPSK, QPSK and 8-PSK modulation

Techniques with $\mathrm{R}=1 / 2$ and constraint length $=7$ 


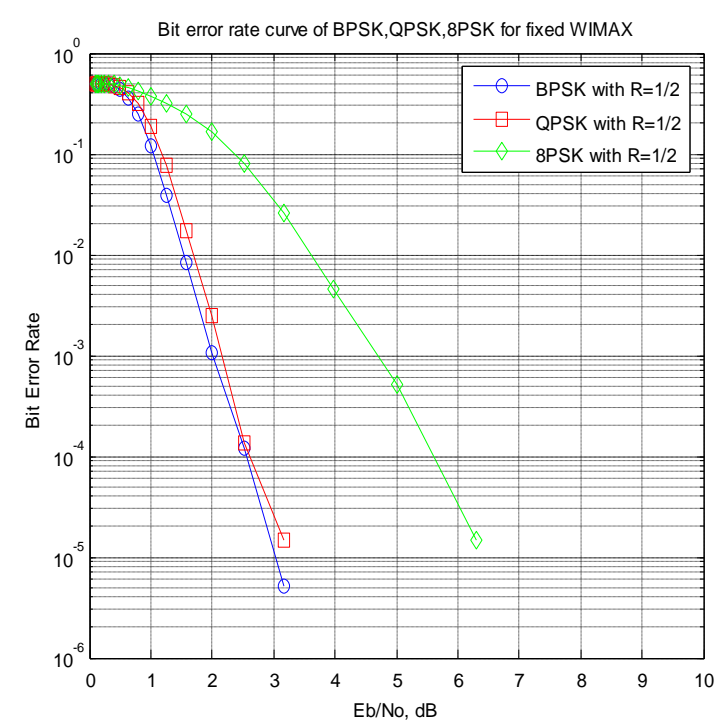

Fig. 2: Demonstrates plot of BER against $E_{b} / N_{0}$ for BPSK, QPSK, 8-PSK modulation techniques

Case2: Considering various levels of QAMs modulation Techniques with $R=1 / 2$ and constraint length $=7$

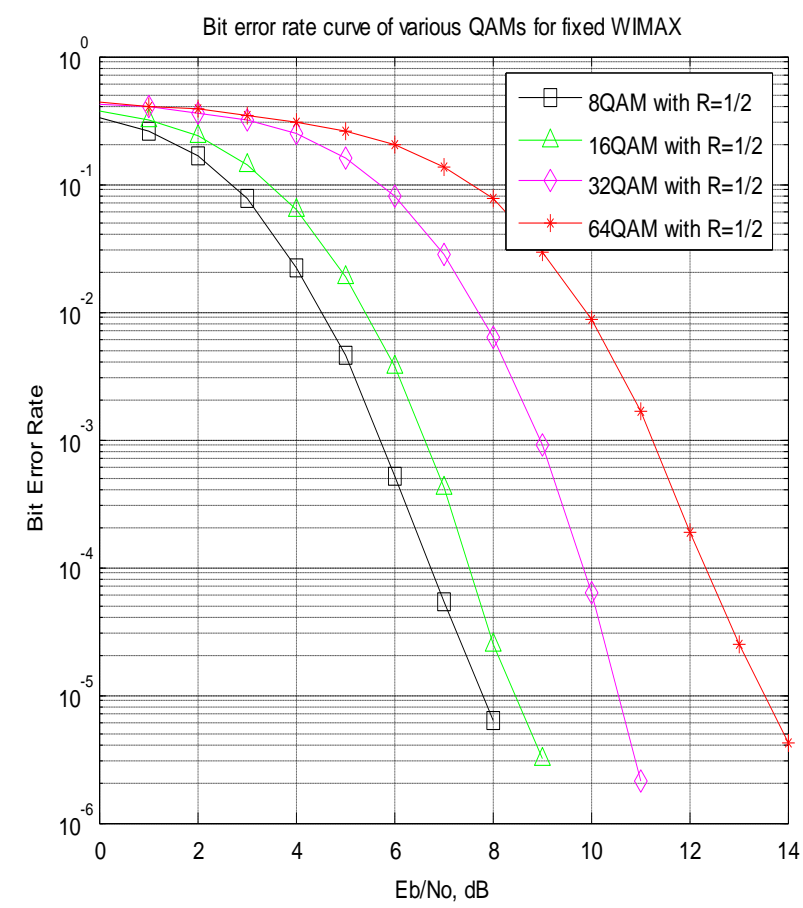

Fig. 3: Demonstrates plot of BER against $E_{b} / \mathbf{N}_{0}$ for various levels of QAMs modulation techniques
Case3: considering various levels of modulation Techniques

$$
\text { with } R=1 / 2 \text { and constraint length }=7
$$

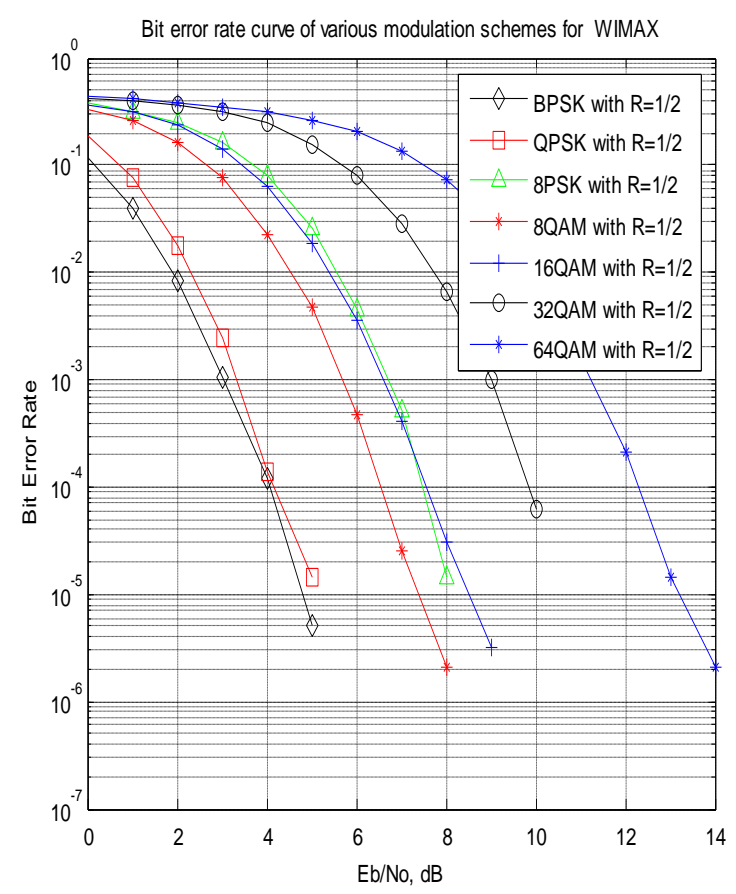

Fig. 4: Demonstrates plot of BER against $E_{b} / N_{0}$ for various digital modulation techniques

Table 2: Values of BER to maintain given $E_{b} / N_{0}$ for various digital modulation techniques

\begin{tabular}{|l|c|c|c|}
\hline $\begin{array}{l}\text { Modulation } \\
\text { scheme }\end{array}$ & $E_{b} / N_{o}=2 d B$ & $E_{b} / N_{o}=6 d B$ & $E_{b} / N_{o}=10 d B$ \\
\hline BPSK & 0.0085 & - & - \\
\hline QPSK & 0.0182 & - & - \\
\hline 8 PSK & 0.2459 & 0.0047 & - \\
\hline 8-QAM & 0.1637 & 0.0004 & - \\
\hline 16-QAM & 0.2342 & 0.0037 & - \\
\hline 32-QAM & 0.3609 & 0.0788 & 0.0001 \\
\hline 64-QAM & 0.3796 & 0.2029 & 0.0087 \\
\hline
\end{tabular}

Fig. 2 shows BER for BPSK, QPSK, 8-PSK modulation techniques against $E_{b} / N_{o}(d b)$ values. Fig. 3 shows BER for QAMs modulation techniques against $\mathrm{E}_{\mathrm{b}} / \mathrm{N}_{\mathrm{o}}(\mathrm{db})$ values. Fig. 4 shows BER for various modulation techniques against $\mathrm{E}_{\mathrm{b}} / \mathrm{N}_{\mathrm{o}}$ (db) values. Depending upon channel conditions adaptive modulation technique[3] can be applied. At the permissible BER of the system, the modulation techniques can be selected for given $E_{b} / N_{o}(d b)$ of channel. Table 2 
depicts the values of $B E R$ to maintain given $E_{b} / N_{o}$ for various digital modulation techniques.

\section{CONCLUSION}

In OFDM system with IEEE 802.16 standard implementation, the various modulation techniques are tested for different channel conditions. The BER performance of system through broadband WiMAX-PHY layer based wireless communication system adopting the Convolutional channel coding and different digital modulation schemes is evaluated. A range of system performance highlights the impact of digital modulations under Convolutional coding. The main conclusions are given below:

- For poor channels conditions (low values of $E_{b} / N_{o}$ $(\mathrm{db})=2 \mathrm{~dB}$ ), BPSK is best modulation techniques with least $\mathrm{BER}=0.0085$. Other alternatives can be QPSK for these conditions.

- For good channels conditions (higher values of $\left.\mathrm{E}_{\mathrm{b}} / \mathrm{N}_{\mathrm{o}}(\mathrm{db})=10 \mathrm{~dB}\right), 32-\mathrm{QAM}$ can be applied.

- From above, we can conclude that for good channel conditions higher level of QAMs give best spectral efficiency and under worst channel conditions i.e. limited value of $\mathrm{E}_{\mathrm{b}} / \mathrm{N}_{\mathrm{o}}$, BPSK and QPSK provide satisfactory results. Thus, we have to use adaptive modulation depending upon channel conditions.

\section{REFERENCES}

[1] Eric Lawrey "The suitability of OFDM as a modulation technique for wireless telecommunications, with a CDMA comparison”, October 1997, pp.25-30.

[2] Guillermo Acosta, "Smart Antenna Research Laboratory", August, 2000.

[3] Eric Phillip Lawrey, "Adaptive Techniques for Multiuser OFDM", December 2001, pp. 127-132.

[4] IEEE Standard 802.16-2005, "Part 16: Air Interface for fixed and mobile broadband wireless access systems, Feb. 2006.

[5] J. El-Najjar, B. Jaumard, C. Assi, "Minimizing Interference in WiMax/802.16 based Mesh Networks with Centralized Scheduling", Global Telecommunications Conference, 2008.pp.1-6.

[6] S. Glisic. "Advanced Wireless Communications, 4G Technology”. John Wiley \& Sons Ltd:Chichester, 2004.

[7] Nuaymi Loutfi, 2007: "WiMAX Technology for Broadband Wireless Access", Wiley, London. 